\title{
Pathologic Findings in Non-Neoplastic Kidney
}

National Cancer Institute

\section{Source}

National Cancer Institute. Pathologic Findings in Non-Neoplastic Kidney. NCI Thesaurus.

Code C159320.

A section header requesting results for the pathologic findings in the non-neoplastic kidney. 